\title{
Scaling, Intermittency and Decay of MHD Turbulence
}

\author{
A. Lazarian and J. Cho (lazarian, cho@astro.wisc.edu) \\ University of Wisconsin-Madison, Dept. of Astronomy
}

\begin{abstract}
We discuss a few recent developments that are important for understanding of MHD turbulence. First, MHD turbulence is not so messy as it is usually believed. In fact, the notion of strong non-linear coupling of compressible and incompressible motions along MHD cascade is not tenable. Alfven, slow and fast modes of MHD turbulence follow their own cascades and exhibit degrees of anisotropy consistent with theoretical expectations. Second, the fast decay of turbulence is not related to the compressibility of fluid. Rates of decay of compressible and incompressible motions are very similar. Third, viscosity by neutrals does not suppress MHD turbulence in a partially ionized gas. Instead, MHD turbulence develops magnetic cascade at scales below the scale at which neutrals damp ordinary hydrodynamic motions. Forth, density statistics does not exhibit the universality that the velocity and magnetic field do. For instance, at small Mach numbers the density is anisotropic, but it gets isotropic at high Mach numbers. Fifth, the intermittency of magnetic field and velocity are different. Both depend on whether the measurements are done in local system of reference oriented along the local magnetic field or in the global system of reference related to the mean magnetic field.
\end{abstract}

Keywords: turbulence, statistics, MHD

\section{What is MHD Turbulence?}

This short review is focused on the recently uncovered basic properties of MHD turbulence ${ }^{1}$. We also briefly deal with recovery of the 3D statistics of turbulent velocity from observations, which is a theoretical problem in itself.

When do we expect fluids to be turbulent? A fluid of viscosity $\nu$ becomes turbulent when the rate of viscous dissipation, which is $\sim \nu / L^{2}$ at the energy injection scale $L$, is much smaller than the energy transfer rate $\sim V_{L} / L$, where $V_{L}$ is the velocity dispersion at the scale $L$. The ratio of the two rates is the Reynolds number $R e=V_{L} L / \nu$. In general, when $R e$ is larger than $10-100$ the system becomes turbulent. Chaotic structures develop gradually as $R e$ increases, and those with $R e \sim$ $10^{3}$ are appreciably less chaotic than those with $R e \sim 10^{8}$. Observed features such as star forming clouds are very chaotic for $R e>10^{8}$. This makes it difficult to simulate realistic turbulence. The currently

\footnotetext{
${ }^{1}$ It is not possible to cite all the important papers in the area of MHD turbulence and turbulent molecular clouds. An incomplete list of the references in a recent review on the statistics of MHD turbulence by Cho, Lazarian \& Vishniac (2003a; henceforth CLV03a) includes about two hundred entries.
}

(c) 2018 Kluwer Academic Publishers. Printed in the Netherlands. 
available 3D simulations containing 512 grid cells along each side can have $R e$ up to $\sim O\left(10^{3}\right)$ and are limited by their grid sizes. Therefore, it is essential to find "scaling laws" in order to extrapolate numerical calculations $\left(\operatorname{Re} \sim O\left(10^{3}\right)\right)$ to real astrophysical fluids $\left(\operatorname{Re}>10^{8}\right)$. We show below that even with its limited resolution, numerics is a great tool for testing scaling laws.

Kolmogorov theory provides a scaling law for incompressible nonmagnetized hydrodynamic turbulence (Kolmogorov 1941). This law provides a statistical relation between the relative velocity $v_{l}$ of fluid elements and their separation $l$, namely, $v_{l} \sim l^{1 / 3}$. An equivalent description is to express spectrum $E(k)$ as a function of wave number $k$ $(\sim 1 / l)$. The two descriptions are related by $k E(k) \sim v_{l}^{2}$. The famous Kolmogorov spectrum is $E(k) \sim k^{-5 / 3}$. The applications of Kolmogorov theory range from engineering research to meteorology (see Monin \& Yaglom 1975) but its astrophysical applications are poorly justified and the application of the Kolmogorov theory can lead to erroneous conclusions (see reviews by Lazarian et al. 2003 and Lazarian \& Yan 2003).

Let us consider incompressible MHD turbulence first ${ }^{2}$. There have long been understanding that the MHD turbulence is anisotropic (e.g. Shebalin et al. 1983). Substantial progress has been achieved recently by Goldreich \& Sridhar (1995; hereafter GS95), who made an ingenious prediction regarding relative motions parallel and perpendicular to magnetic field $\mathbf{B}$ for incompressible MHD turbulence. An important observation that leads to understanding of the GS95 scaling 3 is that magnetic field cannot prevent mixing motions of magnetic field lines if the motions are perpendicular to the magnetic field. Those motions will cause, however, waves that will propagate along magnetic field lines. If that is the case, the time scale of the wave-like motions along the field, i.e. $\sim l_{\|} / V_{A},\left(l_{\|}\right.$is the characteristic size of the perturbation along the magnetic field and $V_{A}=B / \sqrt{4 \pi \rho}$ is the local Alfven speed) will be equal to the hydrodynamic time-scale, $l_{\perp} / v_{l}$, where $l_{\perp}$ is the characteristic size of the perturbation perpendicular to the magnetic field. The mixing motions are hydrodynamic-like ${ }^{4}$ They obey Kolmogorov

\footnotetext{
${ }^{2}$ Traditionally there is insufficient interaction between researchers dealing with compressible and incompressible MHD turbulence. This is very unfortunate, as we will show later that there are many similarities between the properties of incompressible MHD turbulence and those of its compressible counterpart.

3 Here we provide a more intuitive description, while a GS95 presents a more mathematical one.

4 Simulations in Cho, Lazarian \& Vishniac ((2002a, 2003b) that the mixing motions are hydrodynamic up to high order. These motions according to Cho et al. (2003) allow efficient turbulent heat conduction.
} 
scaling, $v_{l} \propto l_{\perp}^{1 / 3}$, because incompressible turbulence is assumed. Combining the two relations above we can get the GS95 anisotropy, $l_{\|} \propto l_{\perp}^{2 / 3}$ (or $k_{\|} \propto k_{\perp}^{2 / 3}$ in terms of wave-numbers). If we interpret $l_{\|}$as the eddy size in the direction of the local magnetic field. and $l_{\perp}$ as that in the perpendicular directions, the relation implies that smaller eddies are more elongated. The latter is natural as it the energy in hydrodynamic motions decreases with the decrease of the scale. As the result it gets more and more difficult for feeble hydrodynamic motions to bend magnetic field lines.

GS95 predictions have been confirmed numerically (Cho \& Vishniac 2000; Maron \& Goldreich 2001; Cho, Lazarian \& Vishniac 2002a, hereafter CLV02a; see also CLV03a); they are in good agreement with observed and inferred astrophysical spectra (see CLV03a). What happens in a compressible MHD? Does any part of GS95 model survives? Literature on the properties of compressible MHD is very rich (see reviews by Pouquet 1999; Cho \& Lazarian 2003b and references therein). Higdon (1984) theoretically studied density fluctuations in the interstellar MHD turbulence. Matthaeus \& Brown (1988) studied nearly incompressible MHD at low Mach number and Zank \& Matthaeus (1993) extended it. In an important paper Matthaeus et al. (1996) numerically explored anisotropy of compressible MHD turbulence. However, those papers do not provide universal scalings of the GS95 type.

The complexity of the compressible magnetized turbulence with magnetic field made some researchers believe that the phenomenon is too complex to expect any universal scalings for molecular cloud research. Alleged high coupling of compressible and incompressible motions is often quoted to justify this point of view (see discussion of this point below).

In what follows we discuss the turbulence in the presence of regular magnetic field which is comparable to the fluctuating one. Therefore for most part of our discussion, we shall discuss results obtained for $\delta V \sim \delta B / \sqrt{4 \pi \rho} \sim B_{0} / \sqrt{4 \pi \rho}$, where $\delta B$ is the r.m.s. strength of the random magnetic field. However, we would argue that our choice is not so restrictive as it may be seen. Indeed, at the scales where the velocity perturbations are much larger than the Alfven velocity, the dynamical importance of magnetic field is small. Therefore we expect that at those scales turbulent motions are close to hydrodynamic ones. At smaller scales where the local turbulent velocity gets smaller than the Alfven speed we believe that our picture will be approximately true. We think that the local magnetic field should act as $B_{0}$, while the small scale perturbations happen in respect to that local field. This reasoning 


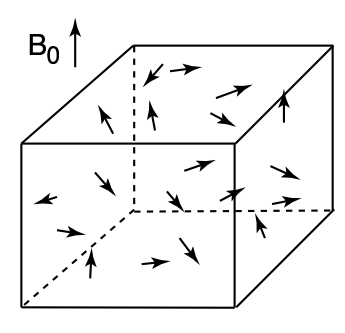

real space

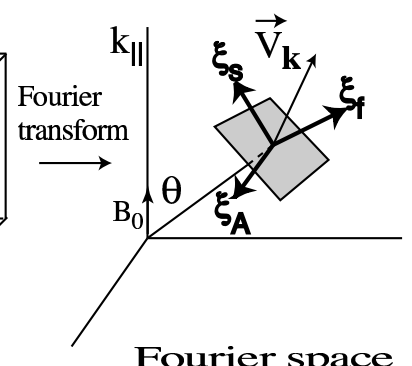

Fourier space

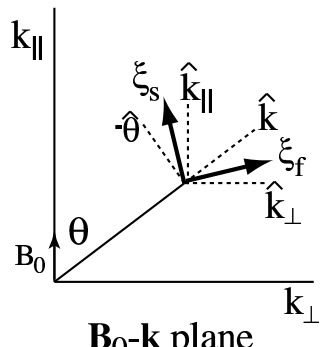

$\mathbf{B}_{0}$-k plane

Figure 1. Separation method. We separate Alfven, slow, and fast modes in Fourier space by projecting the velocity Fourier component $\mathbf{v}_{\mathbf{k}}$ onto bases $\xi_{A}, \xi_{s}$, and $\xi_{f}$, respectively. Note that $\xi_{A}=-\hat{\varphi}$. Slow basis $\xi_{s}$ and fast basis $\xi_{f}$ lie in the plane defined by $\mathbf{B}_{0}$ and $\mathbf{k}$. Slow basis $\xi_{s}$ lies between $-\hat{\theta}$ and $\hat{\mathbf{k}}_{\|}$. Fast basis $\xi_{f}$ lies between $\hat{\mathbf{k}}$ and $\hat{\mathbf{k}}_{\perp}$.

is in agreement with calculations in Cho, Lazarian \& Vishniac (2003b) and Cho \& Lazarian (2003a).

\section{Does the Decay of MHD Turbulence Depend on Compressibility?}

Many astrophysical problems, e.g. the turbulent support of molecular clouds (see review by McKee 1999), critically depends on the rate of turbulence decay. For a long time magnetic fields were thought to be the means of making turbulence less dissipative. Therefore it came as a surprise when numerical calculations by Mac Low et al. (1998) and Stone, Ostriker, \& Gammie (1998) indicated that compressible MHD turbulence decays as fast as the hydrodynamic turbulence. This gives rise to a erroneous belief that it is the compressibility that is responsible for the rapid decay of MHD turbulence.

This point of view has been challenged in Cho \& Lazarian (2002, 2003a, henceforth CL02 and CL03, respectively). In these papers a technique of separating different MHD modes was developed and used (see Fig. 1). This allowed us to follow how the energy was redistributed between these modes.

Should the different MHD modes be strongly coupled when the turbulence is strong? A naive answer is "yes". Indeed, strong turbulence implies strong field line wondering. This mixes up Alfven and fast modes. In addition, one can show through calculations that the magnetic non-linearities result in the drainage of energy from Alfvenic cascade. However, a remarkable feature of the GS95 model is that Alfven perturbations cascade to small scales over just one wave period, which gets shorter and shorter as we move along the cascade. The 
competing effects coupling different modes usually require more time ${ }^{5}$. We note that as the consequence of this reasoning we should assume that the properties of the Alfvenic cascade (incompressible cascade!) should not strongly depend on the sonic Mach number.

Are the arguments above correct? The generation of compressible motions (i.e. radial components in Fourier space) from Alfvenic turbulence is a measure of mode coupling. How much energy in compressible motions is drained from Alfvenic cascade? According to closure calculations (Bertoglio, Bataille, \& Marion 2001; see also Zank \& Matthaeus 1993), the energy in compressible modes in hydrodynamic turbulence scales as $\sim M_{s}^{2}$ if $M_{s}<1$. CL03 conjectured that this relation can be extended to MHD turbulence if, instead of $M_{s}^{2}$, we use $\sim(\delta V)_{A}^{2} /\left(a^{2}+V_{A}^{2}\right)$. (Hereinafter, we define $V_{A} \equiv B_{0} / \sqrt{4 \pi \rho}$, where $B_{0}$ is the mean magnetic field strength.) However, since the Alfven modes are anisotropic, this formula may require an additional factor. The compressible modes are generated inside the so-called Goldreich-Sridhar cone, which takes up $\sim(\delta V)_{A} / V_{A}$ of the wave vector space. The ratio of compressible to Alfvenic energy inside this cone is the ratio given above. If the generated fast modes become isotropic (see below), the diffusion or, "isotropization" of the fast wave energy in the wave vector space increase their energy by a factor of $\sim V_{A} /(\delta V)_{A}$. This results in

$$
\frac{(\delta V)_{\mathrm{rad}}^{2}}{(\delta V)_{A}^{2}} \sim\left[\frac{V_{A}^{2}+a^{2}}{(\delta V)_{A}^{2}} \frac{(\delta V)_{A}}{V_{A}}\right]^{-1},
$$

where $(\delta V)_{\text {rad }}^{2}$ and $(\delta V)_{A}^{2}$ are energy of compressible and Alfven modes, respectively. Eq. (1) suggests that the drain of energy from Alfvenic modes is marginal along the cascade ${ }^{6}$ when the amplitudes of perturbations are weak. Results of calculations shown in Fig. 2 support the theoretical predictions.

We may summarize this issue in the following way. For the incompressible motions to decay fast, there is no requirement of coupling with compressible motions ${ }^{7}$. The marginal coupling of the compressible and incompressible modes allows us to study these modes separately.

\footnotetext{
${ }^{5}$ This reasoning shows that at the energy injection scale when $\delta B \sim B_{0}$ the coupling between the modes is appreciable.

6 The marginal generation of compressible modes is in agreement with earlier studies by Boldyrev, Nordlund, \& Padoan (2002) and Porter, Pouquet, \& Woodward (2002), where the velocity was decomposed into a potential component and a solenoidal component. A recent study by Vestuto, Ostriker \& Stone (2003) is also consistent with this conclusion.

7 The reported (see Mac Low et al. 1998) decay of the total energy of turbulent motions $E_{\text {tot }}$ follows $t^{-1}$ which can be understood if we account for the fact that the energy is being injected at the scale smaller than the scale of the system. Therefore
} 

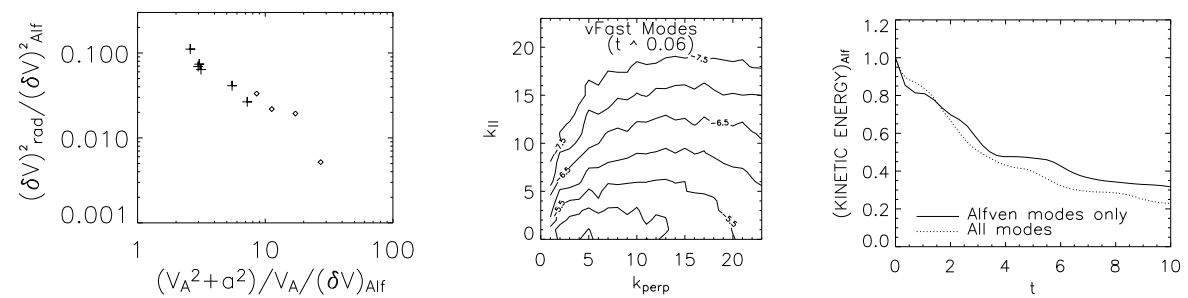

Figure 2. Mode coupling studies. (a)left: Square of the r.m.s. velocity of the compressible modes. We use $144^{3}$ grid points. Only Alfven modes are allowed as the initial condition. "Pluses" are for low $\beta$ cases $(0.02 \leq \beta \leq 0.4)$. "Diamonds" are for high $\beta$ cases $(1 \leq \beta \leq 20)$. (b) middle: Generation of fast modes. Snapshot is taken at $\mathrm{t}=0.06$ from a simulation (with $144^{3}$ grid points) that started off with Alfven modes only. Initially, $\beta$ (ratio of gas to magnetic pressure, $P_{g} / P_{\text {mag }}$ ) $=0.2$ and $M_{s}$ (sonic Mach number) 1.6. (c)right: Comparison of decay rates. Decay of Alfven modes is not much affected by other (slow and fast) modes. We use $216^{3}$ grid points. Initially, $\beta=0.02$ and $M_{s} \sim 4.5$ for the solid line and $M_{s} \sim 7$ for the dotted line. Note that initial data are, in some sense, identical for the solid and the dotted lines. The sonic Mach number for the solid line is smaller because we removed fast and slow modes from the initial data before the decay simulation. For the dotted line, we did not remove any modes from the initial data. From CL03.

\section{What are the scalings for velocity and magnetic field?}

Some hints about effects of compressibility can be inferred from the GS95 seminal paper. More discussion was presented in Lithwick \& Goldreich (2001), which primary deals with electron density fluctuations in the regime of high $\beta$, i.e. $\left(\beta \equiv P_{\text {gas }} / P_{\text {mag }} \gg 1\right)$. As the incompressible regime corresponds to $\beta \rightarrow \infty$, so it is natural to expect that for $\beta \gg 1$ the GS95 picture would persist. Lithwick \& Goldreich (2001) also speculated that for low $\beta$ plasmas the GS95 scaling of slow modes may be applicable. A detailed study of compressible mode scalings is given in CL02 and CL03.

Our considerations above about the mode coupling can guide us in the discussion below. Indeed, if Alfven cascade evolves on its own, it is natural to assume that slow modes exhibit the GS95 scaling. Indeed, slow modes in gas pressure dominated environment (high $\beta$ plasmas) are similar to the pseudo-Alfven modes in incompressible regime (see GS95; Lithwick \& Goldreich 2001). The latter modes do follow the GS95 scaling. In magnetic pressure dominated environments or low $\beta$ plasmas, slow modes are density perturbations propagating with the

some energy originally diffuses to larger scales through the inverse cascade. Our calculations (Cho \& Lazarian, unpublished), stimulated by illuminating discussions with Chris McKee, show that if this energy transfer is artificially prevented by injecting the energy on the scale of the computational box, the scaling of $E_{\text {tot }}$ becomes closer to $t^{-2}$. 


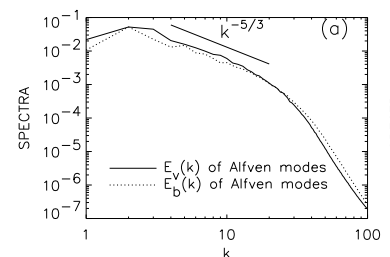

(a)

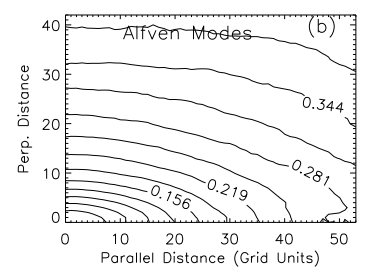

(b)

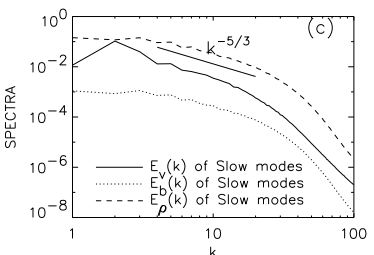

(c)

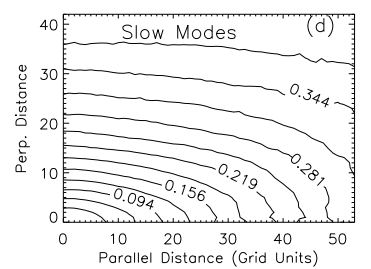

(d)

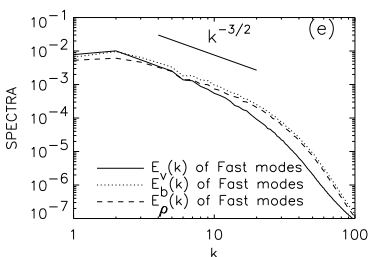

(e)

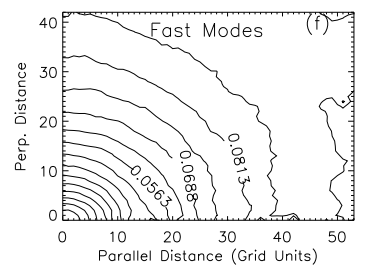

(f)

Figure 3. $M_{s} \sim 2.2, M_{A} \sim 0.7, \beta \sim 0.2$, and $216^{3}$ grid points. (a) Spectra of Alfvén modes follow a Kolmogorov-like power law. (b) Eddy shapes (contours of same second-order structure function, $S F_{2}$ ) for velocity of Alfvén modes shows anisotropy similar to the GS95 $\left(r_{\|} \propto r_{\perp}^{2 / 3}\right.$ or $\left.k_{\|} \propto k_{\perp}^{2 / 3}\right)$. The structure functions are measured in directions perpendicular or parallel to the local mean magnetic field in real space. We obtain real-space velocity and magnetic fields by inverse Fourier transform of the projected fields. (c) Spectra of slow modes also follow a Kolmogorov-like power law. (d) Slow mode velocity shows anisotropy similar to the GS95. We obtain contours of equal $S F_{2}$ directly in real space without going through the projection method, assuming slow mode velocity is nearly parallel to local mean magnetic field in low $\beta$ plasmas. (e) Spectra of fast modes are compatible with the IK spectrum. (f) The magnetic $S F_{2}$ of fast modes shows isotropy. From CL02

sound speed $a$ parallel to the mean magnetic field. Those perturbations are essentially static for $a \ll V_{A}$. Therefore Alfvenic turbulence is expected to mix density perturbations as if they were passive scalar. This also induces the GS95 spectrum.

The fast waves in low $\beta$ regime propagate at $V_{A}$ irrespectively of the magnetic field direction. In high $\beta$ regime, the properties of fast modes are similar, but the propagation speed is the sound speed $a$. Thus the mixing motions induced by Alfven waves should marginally affect the fast wave cascade. It is expected to be analogous to the acoustic wave cascade and hence be isotropic.

Results of numerical calculations from Cho \& Lazarian (CL03) for magnetically dominated media similar to that in molecular clouds are shown in Fig. 3. They support theoretical considerations above. 

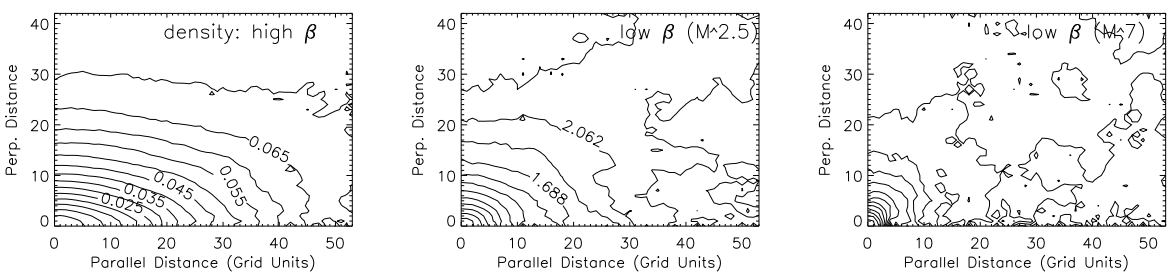

Figure 4. left panel: Mach number is 0.35 , central panel: Mach number is 2.3 right panel: Mach number is 7. The figures are from CL03

\section{What is the scaling of density?}

Density at low Mach numbers follow the GS95 scaling when the driving is incompressible (CL03). However, CL03 showed that this scaling substantially changes for high Mach numbers. Fig. 4 shows that at high Mach numbers density fluctuations get isotropic. Moreover, our present studies confirm the CL03 finding that the spectrum of density gets substantially flatter than the GS95 one (see also Cho \& Lazarian 2004). Note, that a model of random shocks would produce a spectrum steeper than the GS95 one. A possible origin of the flat spectrum is the superAlfvenic perturbations created by fast modes within density perturbations originated from slow modes. This particular regime is clearly identified in a review by Cho, Lazarian \& Vishniac (2003) (see Fig. 9). It may also be related to the regime of superAlfvenic turbulence discussed e.g. in Norlund \& Podoan (2003). However, alternative explanations of the shallow density fluctuations exist and our current work should clarify which process is actually responsible for the unusual density scaling that we observe.

\section{How do neutrals affect MHD turbulence?}

An obvious effect of neutrals is that they do not follow magnetic field lines and thus produce viscosity.

In hydrodynamic turbulence viscosity sets a cutoff, with an exponential suppression of motion on smaller scales. Below the viscous cutoff the kinetic energy contained in a wavenumber band is dissipated at that scale, instead of being transferred to smaller scales. This means the end of the hydrodynamic cascade, but in MHD turbulence this is not the end of magnetic structure evolution. If viscosity is much larger than resistivity, there is a broad range of scales where viscosity is important but resistivity is not. Over these scales magnetic field will be stretched by turbulent motions at larger scales, with the shear from the smallest 

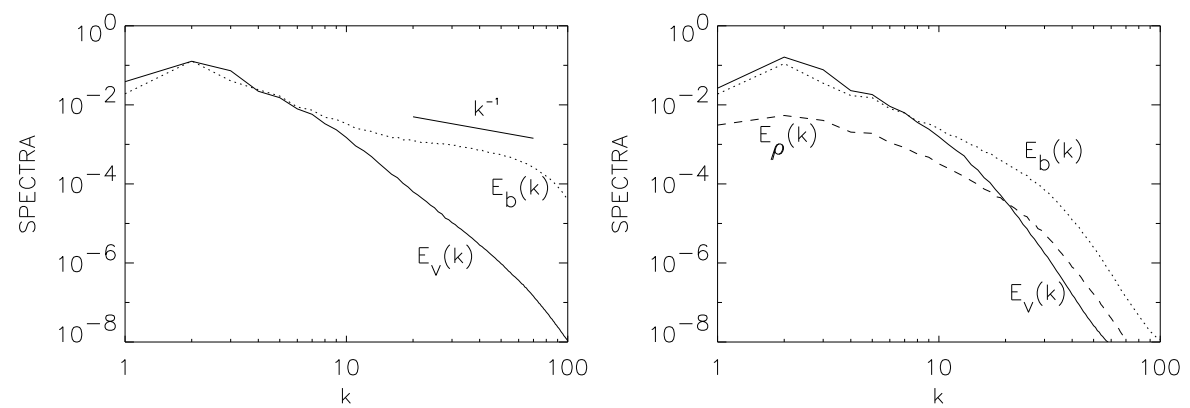

Figure 5. Viscous damped regime (viscosity $>$ magnetic diffusivity). Due to large viscosity, velocity damps after $k \sim 10$. (a) Left: Incompressible case with $384^{3}$ grid points. Magnetic spectra show a shallower slope $\left(E_{b}(k) \propto k^{-1}\right)$ below the velocity damping scale. We achieve a very small magnetic diffusivity through the use hyper-diffusion. From Cho, Lazarian, \& Vishniac (2002b). (b) Right: Compressible case with $216^{3}$ grid points. Magnetic and density spectra show structures below the velocity damping scale at $k \sim 10$. The structures are less obvious than the incompressible case because it is relatively hard to achieve very small magnetic diffusivity in the compressible run. From CL03.

undamped eddies being most important. Indeed, this new regime of turbulence has been discovered (see Fig. 5)!

A theoretical model for this new regime is given in Lazarian, Vishniac, \& Cho (2004; hereafter LVC04). It predicts the spectrum $E(k) \sim$ $k^{-1}$ for magnetic field and the spectrum $\sim k^{-4}$ for kinetic energy. The actual measurements that got somewhat steeper spectra were explained in LVC04 as the consequences of a small inertial range available.

An important prediction in LVC04 is that the intermittency of magnetic structures increases with the decrease of the scale. This prediction was confirmed by numerical simulations in Cho, Lazarian \& Vishniac (2003b), which showed that the filling factor of magnetic field was decreasing with the increase of the wavenumber.

The effect of neutrals does not amounts only to emergence of the viscosity-damped regime of MHD turbulence. Below we describe some other effects.

First of all, it is clear that whether ions and neutrals act as one fluid depends on whether the eddy turnover rate $t_{\text {eddy }}^{-1} \sim k v_{k}$ is longer or shorter than the rate $t_{n i}^{-1}$ of neutral-ion collisions. If $t_{e d d y}^{-1}>t_{n i}^{-1}$, neutrals decouple from ions and develop hydrodynamic Kolmogorov-type cascade. Indeed, the damping rate for those hydrodynamic motions $t_{n i}^{-1}$ and below the decoupling scale the hydrodynamic motions evolve without much hindrance from magnetic field. Magnetic fields with the entrained ions develop the viscosity-damped MHD cascade until ionneutral collisional rate gets longer than the dynamical rate of the 
intermittent magnetic structures. After that the turbulence reverts to its normal MHD cascade which involves only ions.

If $t_{e d d y}^{-1}<t_{n i}^{-1}$ up to the scale at which neutral viscosity damps turbulent motions, the viscosity-damped regime emerges at the scale where kinetic energy associated with turbulent eddies is dissipated. Similarly to the earlier case when the when ion-neutral collisions get insufficient to preserve pressure confinement of the small scale magnetic filaments, outbursts of ordinary ionic MHD turbulence will take place. The turbulence will be intermittent both in time and space because of the disparity of time scales at which turbulence evolves in the viscositydamped and free ionic MHD regimes. Those predictions should be tested with a two fluid MHD code.

\section{What is the intermittency of MHD turbulence?}

Power spectra do not uniquely characterize turbulence. Very different random processes may have the same power spectra. One way to break this degeneracy is to use higher order structure functions.

The p-th order (longitudinal) velocity structure function $S F_{p}$ and scaling exponents $\zeta(p)$ are defined as

$$
S F_{p}(\mathbf{r}) \equiv\left\langle|[\mathbf{v}(\mathbf{x}+\mathbf{r})-\mathbf{v}(\mathbf{x})] \cdot \hat{\mathbf{r}}|^{p}\right\rangle \propto r^{\zeta(p)},
$$

where the angle brackets denote averaging over $\mathbf{x}$.

The scaling relations suggested by She \& Leveque (1994) related $\zeta(p)$ to the scaling of the velocity $v_{l} \sim l^{1 / g}$, the energy cascade rate $t_{l}^{-1} \sim l^{-x}$, and the co-dimension of the dissipative structures $C$ :

$$
\zeta(p)=\frac{p}{g}(1-x)+C\left(1-(1-x / C)^{p / g}\right) .
$$

For incompressible turbulence these parameters are $g=3, x=2 / 3$, and $C=2$, implying that dissipation happens over $1 \mathrm{D}$ structures (e.g. vortices).

Muller \& Biskamp (2000) applied this model to MHD turbulence and attracted the attention of the MHD researchers to this tool. CLV03 discovered that the magnetic field and velocity has different $\zeta: \zeta^{\text {magnetic }}<$ $\zeta^{\text {velocity }}$, which means that magnetic field is more intermittent than velocity. In addition, in CLV03 we found that the value of $C$ changes depending whether we use local frame of reference which is perpendicular to magnetic field lines or a global one related to the large scale mean magnetic field. These complications make us wonder whether the parameters of the She-Leveque model have the same clear interpretation in MHD case as they have in hydro. 

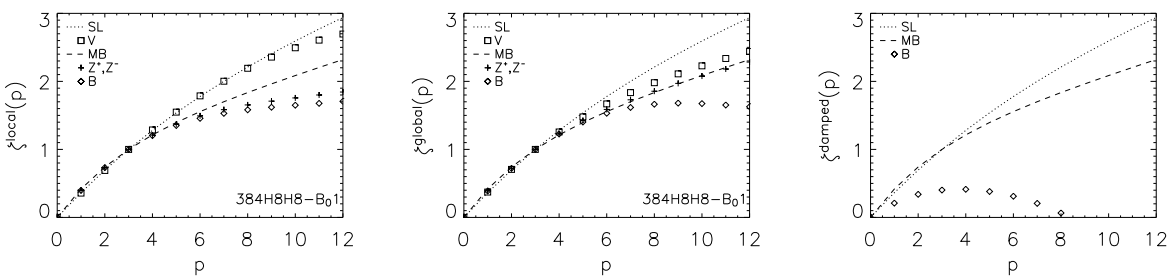

Figure 6. left panel:Normalized structure function exponents in perpendicular directions in the local frame. The velocity exponents show a scaling similar to the She-Leveque model. The magnetic field shows a different scaling. central panel:Normalized structure function exponents in the global frame. Note that the result for $z^{ \pm}$is very similar to the Müller-Biskamp model. right panel: Magnetic structure function exponents, $\zeta(p)$, in the local frame (not normalized). The observed scaling exponents are at least close to the expected asymptote $\zeta(p)=0$. Figures are from CLV03

\section{Summary}

1. MHD turbulence is not a mess. Scaling relations for its modes have been established recently.

2. Fast decay of MHD turbulence is not due to strong coupling of compressible and incompressible motions. The transfer of energy from Alfven to compressible modes is small. The Alfven mode develops on its own and decays fast.

3. MHD turbulence does not vanish at the viscous scale, provided that the fluid viscosity is much larger than resistivity. Instead, a new regime of turbulence in which magnetic energy cascade is driven by larger scale turbulent motions develops.

4. Density fluctuations follow the scaling of Alfvenic part of the cascade only at small Mach numbers. At large Mach numbers the density field gets isotropic and has a shallow spectrum.

5. The intermittency of magnetic field is larger than that of the velocity field. The physical meaning of the She-Leveque dimensions is not straightforward as it does depend on whether the measurements are done in local or global reference frame.

Acknowledgments $W$ e acknowledge NSF grant AST 0307869 and the NSF Center for Magnetic Self-Organization in the Laboratory and Astrophysical Plasmas.

\section{References}

Bertoglio, J.-P., Bataille, F., \& Marion, J.-D. 2001, Phys. Fluids, 13, 290

Boldyrev, S., Nordlund, Å., \& Padoan P. 2002a, Phys. Rev. Lett. 89, 031102

Cho, J., Lazarian, A. 2002a, Phy. Rev. Lett., 88, 245001 (CL02) 
Cho, J., Lazarian, A. 2003a, MNRAS, 345, 325 (CL03)

Cho, J., Lazarian, A. 2003b, preprint (astro-ph/0301462)

Cho, J., Lazarian, A. 2004, preprint

Cho, J., Lazarian, A., Honein, A., Knaepen, B., Kassinos, S., \& Moin, P. 2003, ApJ, 589, L77

Cho, J., Lazarian, A., \& Vishniac, E. 2002a, ApJ, 564, 291 (CLV02a)

Cho, J., Lazarian, A., \& Vishniac, E. 2002b, ApJ, 566, L49 (CLV02b)

Cho, J., Lazarian, A., \& Vishniac, E. 2003a, in Turbulence and Magnetic Fields in Astrophysics, eds. E. Falgarone \& T. Passot (Springer LNP), p56 (astroph/0205286) (CLV03a)

Cho, J., Lazarian, A., \& Vishniac, E. 2003b, ApJ, 595, 812

Cho, J. \& Vishniac, E. 2000, ApJ, 539, 273

Goldreich, P. \& Sridhar, S. 1995, ApJ, 438, 763 (GS95)

Higdon, J. C. 1984, ApJ, 285, 109

Kolmogorov, A. 1941, Dokl. Akad. Nauk SSSR, 31, 538

Lazarian, A., Petrosian, V., Yan, H., \& Cho, J. 2003, in press (astro-ph/0301181)

Lazarian, A. \& Vishniac, E. T., \& Cho, J. 2004, ApJ, 603, 180 (LVC04)

Lazarian, A. \& Yan, H. 2003, in "Astrophysical Dust" eds. A. Witt \& B. Draine, APS, in press

Lithwick, Y. \& Goldreich, P. 2001, ApJ, 562, 279

Mac Low, M.-M., Klessen, R., Burkert, A., \& Smith, M. 1998, Phys. Rev. Lett., 80, 2754

Maron, J. \& Goldreich, P. 2001, ApJ, 554, 1175

Matthaeus, W. H. \& Brown, M. R. 1988, Phys. Fluids, 31(12), 3634

Matthaeus, W. H., Ghosh, S., Oughton, S., \& Roberts, D. A. 1996, J. Geophysical Res., 101(A4), 7619

McKee C. 1999 The Origin of Stars and Planetary Systems. Eds. Charles J. Lada and Nikolaos D. Kylafis. Kluwer, p.29

Monin, A.S., \& Yaglom, A.M. 1975, Statistical Fluid Mechanics: Mechanics of Turbulence, vol. 2, The MIT Press

Müller, W.-C. \& Biskamp, D. 2000, Phys. Rev. Lett., 84(3), 475

Norlund, A., Podoan, P. 2003, in Turbulence and Magnetic Fields in Astrophysics, eds. E. Falgarone \& T. Passot (Springer LNP), p.271

Porter, D., Pouquet, A., \& Woodward, P. 2002, Phys. Rev. E, 66, 026301

Pouquet, A. 1999, in Interstellar Turbulence, p.87

Shebalin, J. V., Matthaeus, W. H., \& Montgomery, D. C. 1983, J. Plasma Phys., 29,525

She, Z., \& Leveque, E. 1994, Phys. Rev. Lett., 72, 336

Stone, J., Ostriker, E., \& Gammie, C. 1998, ApJ, 508, L99

Vestuto, J. G., Ostriker, E. C., \& Stone, J. M. 2003, ApJ, 590, 858

Zank, G. P. \& Matthaeus, W. H. 1993, Phys. Fluids A, 5(1), 257 